\title{
Antibiotic Resistance and Upper Respiratory Infections
}

\author{
Juan Antonio Lugo Machado* \\ Surgeon Specialist in Otorhinolaryngology Department of Otolaryngolgoy, Centro Médico Nacional del Noroeste, México
}

*Corresponding author: Lugo Machado Juan Antonio, Surgeon Specialist in Otorhinolaryngology, Sub specialist in Pediatric Otolaryngology, Assigned Physician of Otolaryngology, National Medical Center of the Northwest, IMSS, Cd. Obregón, Sonora, Mexico.

Received Date: August 10, 2018

Published Date: September 11, 2018

\section{Resistance to antibiotics and upper respiratory infections}

Resistance to antibiotics are part of the threats to global health today, resistance can affect anyone in the world who is facing an infectious process of any kind. This phenomenon is a natural process, in any living being that seeks to maintain itself, reproduce and perpetuate itself, although the improper use of antibiotics is accelerating the process.

Increasingly, infections with resistance to antibiotics are registered, for example, pneumonia, tuberculosis, gonorrhea whose treatment becomes more difficult due to the loss of efficacy of antibiotics.

This process of resistance generates higher healthcare costs, with prolongation of hospital stays, increases medical costs and increases mortality.

The rules for the judicious use of antibiotics in daily clinical practice should not only be left on paper, their application should be supervised, and these rules should be maintained, in order to guarantee the correct use of them.

Clinical practice guidelines are part of the educational process for us physicians, however their use is not limited, at least in developing countries such as Mexico and Latin America.

\section{Prevention and control}

Resistance to antibiotics is accelerated by the improper and abusive use of these drugs and the deficiencies in the prevention and control of infections. Measures can be taken at all levels of society to reduce the impact of this phenomenon and limit its spread.

Among the measures to prevent antimicrobial resistance, in addition to continuing medical education with dissemination of standards for health professionals, must have programs of education to the population to reduce self-medication, as the rules of prescription of antibiotics is overrun by private institutions such as pharmacies, which in a way "require" doctors to prescribe medicines, including antibiotics, although patients do not need it, focusing this situation in my country as Mexico and other Latin American countries, where rules and regulations are broken by economic interests and corruption.

\section{Scope of the problem}

Antibiotic resistance is increasing worldwide to dangerous levels. New mechanisms of resistance are appearing and spreading every day around the planet that jeopardize our ability to treat common infectious diseases. A growing number of infections, such as pneumonia, tuberculosis, septicemia, gonorrhea or foodborne diseases, are increasingly difficult - and sometimes impossible - to treat, as antibiotics lose effectiveness.

\section{Upper Respiratory Tract Infections}

At present antibiotics are abused for upper respiratory tract infections, considering that around $90 \%$ are of viral origin and that it only requires medication for symptoms, nowadays they are misused, both by first-contact physicians, such as self-medication by the patient. The different organisms and institutions at the international level seek to generate greater diffusion about the criteria that a patient with a probability of bacterial infection need to meet, thus justifying the prescription of antibiotics.

In this small review you will find the criteria for the rational use of antibiotics in patients with throat infection of bacterial origin.

Upper respiratory tract infections are the main reasons for medical consultation, and although $90 \%$ of them are viral, $10 \%$ are bacterial and require the use of antibiotics.

The tendency to use antimicrobials indiscriminately by selfmedication or poor medical prescription has encouraged bacterial 
resistance. Because of this tendency and to justify the use of antibiotics by medical prescription, clinical criteria have been established with scientific support, which should be applied by the first contact physicians.

Currently, the use of antibiotics has been associated with the reduction of sore throat symptoms and may reduce the incidence of complications in patients with throat infections caused by group A beta hemolytic streptococcus; Both the American Society of Infectious Diseases (IDSA) and the American Heart Association (AHA) recommend antibiotics only for the group of confirmed streptococcal pharyngitis, either by rapid test of streptococcus or by culture of pharyngeal exudate in adults and children [1].

The preferred antibiotics are penicillin for 10 days or benzathine penicillin G, a single intramuscular dose; however, in case of allergy to said medication, clindamycin is prescribed for 10 days or azithromycin for 5 days; These are the antibiotics recommended by the Center for Disease Control and Prevention (CDC) with evidencebased guidelines.

In this context, antibiotics should be limited to patients with pharyngitis and / or tonsillitis who are more likely to have a group A beta-hemolytic Streptococcus infection; these treatment recommendations are for adults with pharyngitis based on the Centor score; 4 criteria; history of fever, presence of exudate in tonsil, presence of anterior cervical lymphadenopathy or absence of cough [2].

If you meet all 4 criteria, empirical treatment should be established; if they meet 3 , the empirical treatment can be started or the antigen rapid test can be carried out, initiating treatment only in case of being positive; if they meet only 2 criteria, the rapid antigen tests must be carried out; antibiotic treatment is indicated only if the test is positive, or symptomatic treatment if the test is negative; if only one or no criteria is met, only symptomatic treatment is given, without antibiotics.

In fact, Dr. Warren J. McIsaac wrote an article in which he modified Centor's criteria, adding one more, which was the patient's age [3]. The Centor criteria modified by Mclsaac were: Temperature $>38^{\circ} \mathrm{C}$, tonsillar exudate, absence of cough, painful cervical cervical adenopathies; Age: 3-4 years, 15-44 years; all, when present, add a point, while the age $>45$ years left one point. Currently penicillin and erythromycin in patients allergic to penicillin are still recommendations made in Australia and New Zealand, very similar.

\section{Conclusion}

The prescription of antibiotics for respiratory tract infections should be justified; At present, there are criteria that support us in clinical practice and thereby restrict the use of antibiotics in patients who require it, reducing its indiscriminate use and bacterial resistance.
The treatment approach includes the prescription of empirical antibiotic in patients most likely to have a streptococcal infection; even, the North American guides backed by organisms as important as the CDC, AHA or IDSA, tend to recommend the empirical use, and antibiotics in the cases that fulfill the mentioned criteria, this to avoid their indiscriminate use [4].

Nowadays penicillin and erythromycin in patients allergic to penicillin are still recommendations made in Australia and New Zealand, very similar to the CDC guidelines.

If urgent action is not taken, the world is heading for a postantibiotic era in which many common infections and minor injuries will again be life-threatening.

In response to the WHO bacterial resistance, a World Plan of Action on Antimicrobial Resistance, including Resistance to Antibiotics, was approved by a World Health Assembly in May 2015 [5]. Its purpose is to ensure that infectious diseases can continue to be prevented and treated through effective and safe drugs.

The action plan includes five strategic objectives: Improve awareness and knowledge on antimicrobial resistance; strengthen surveillance and investigation; reduce the incidence of infections; optimize the use of antimicrobial drugs; ensure that sustainable investments are made in the fight against antimicrobial resistance.

The Secretary General of the United Nations has established the interagency coordination group on antimicrobial resistance in order to improve communication among international organizations and ensure effective global action against this threat to health security.

\section{References}

1. Choby BA (2009) Diagnosis and treatment of streptococcal pharyngitis. Am Fam Physician 79(5): 383-390.

2. Gerber MA, Baltimore RS, Eaton CB, Gewitz M, Rowley AH, et al. (2009) Prevention of rheumatic fever and diagnosis and treatment of acute streptococcal pharyngitis: a scientific statement from the American Heart Association rheumatic fever, endocarditis, and Kawasaki Disease Committee of the council on cardiovascular disease in the young, the Interdisciplinary Council on Functional Genomics and Translational Biology, and the Interdisciplinary Council on quality of care and outcomes research: endorsed by the American Academy of Pediatrics. Circulation 119(11):1541-1551.

3. McIsaac WJ, Kellner JD, Aufricht P, Vanjaka A, Low DE (2004) Empirical validation of guidelines for the management of pharyngitis in children and adults. JAMA 291(13):1587-1595.

4. Shulman ST, Bisno AL, Clegg HW, Gerber MA, Kaplan EL, et al. (2012) Clinical practice guideline for the diagnosis and management of group A streptococcal pharyngitis: update by the infectious diseases society of America. Clin Infect Dis 55(10): 1279-1282.

5. WHO (2018) Antibiotic resistance, World Health Organization, Geneva, Switzerland. 